ISSN 2215-3535 http://revistas.ucr.ac.cr/index.php/actualidades

Recibido: 04 de octubre de 2018 DOI: $10.15517 /$ ap.v33i127.34778

Aceptado: 9 de mayo de 2019

\title{
Cuestionario de Emociones y Creencias acerca de la Alimentación y el Peso (CECAP)
}

\section{Questionnaire of Emotions and Beliefs Regarding Diet and Weight (CECAP)}

\author{
Cecilia Silva ${ }^{1}$ \\ Karla Edith González Alcántara² \\ Universidad Nacional Autónoma de México, Facultad de Psicología, México
}

\begin{abstract}
Resumen. Objetivo. Construir y valorar las propiedades psicométricas de un instrumento que permita operacionalizar las emociones y las creencias que elicitan el consumo de alimentos. Participantes. De manera no probabilística e intencional participaron 1833 hombres (49.9\%) y mujeres (50.1\%), de entre 14 y 18 años $(M$ $=15.43, D T=.93)$. El $95.4 \%$ de los participantes se encontraban estudiando el nivel medio superior y el $4.6 \%$ iniciaba sus estudios de nivel superior en escuelas públicas de la Ciudad de México. Método. Se probó la calidad del instrumento desde la Teoría Clásica de los Test. Solo se aplicó el instrumento realizado ex profeso. Resultados. Se obtuvo un instrumento válido y confiable con tres dimensiones: creencias erróneas acerca del control de peso, experimentación de emociones agradables al comer e importancia que se le otorga al peso corporal.
\end{abstract}

Palabras clave. Joven, México, psicometría, método de medición.

Abstract. Objective. To build and assess the psychometric properties of an instrument that allows the emotions and beliefs elicited by food consumption to be operationalized. Participants. 1833 men (49.9\%) and women (50.1\%), between 14 and 18 years old $(M=15.43, S D=.93)$ participated in this study in a non-probabilistic and intentional way. $95.4 \%$ of the participants were studying at high school level, and $4.6 \%$ began their higher education in public schools in Mexico City. Method. The quality of the instrument was tested according to the Classical Test Theory. Only the purposely-made instrument was implemented. Results. A valid and reliable instrument was obtained, with three dimensions: erroneous beliefs about weight control, experiencing of pleasant emotions when eating, and importance given to body weight.

Keywords. Youth, Mexico, psychometrics, measuring methods.

${ }^{1}$ Cecilia Silva. División de Investigación y Posgrado, Facultad de Psicología, Universidad Nacional Autónoma de México, México. Dirección postal: Av. Universidad 3004, Edificio E, Cubículo 208, Col. Copilco-Universidad, Delegación Coyoacán, C.P. 04510, Ciudad de México. E-mail: csilva@posgrado.unam.mx

${ }^{2}$ Karla Edith González Alcántara. División de Investigación y Posgrado, Facultad de Psicología, Universidad Nacional. E-mail: karlaedith@comunidad.unam.mx

\section{@ $\odot \Theta \Theta$}

Esta obra está bajo una licencia de Creative Commons Reconocimiento-NoComercial-SinObraDerivada 4.0 Internacional. 


\section{Introducción}

La conducta alimentaria está determinada en gran medida por los modelos socioculturales (Rodgers, 2016), las creencias del efecto de los alimentos sobre el cuerpo (Bublitz, Peracchio, \& Block, 2010), por el contexto en el que ocurre la alimentación (Cruwys, Bevelander, \& Hermans, 2015; Sinha, 2016) y por las emociones (Van Strien, Donker, \& Ouwens, 2016).También es cierto que las experiencias tempranas (Howe, Anderson, \& Dewhurst, 2017), particularmente la forma en que la madre se alimenta (Dejesusa et al.; 2018) y las normas sociales (Lally, Bartle, \& Wardle, 2011) influyen en los patrones de consumo de alimentos.

Las creencias, por otra parte, afectan las actitudes de la gente y, por tanto, su conducta (Geraearts et al.; 2008). Particularmente, las creencias falsas o erróneas, acerca del peso y la alimentación podrían conducir a la emisión de conductas con consecuencias tales como la restricción alimentaria o la sobre alimentación, mismas que pueden conducir al sobrepeso, la obesidad o la malnutrición.

En años recientes también se ha establecido la relación entre los estados emocionales y la ingesta de alimentos. Por ejemplo, Ramos, González y Silva (2016) encontraron que las personas con sobrepeso y obesidad tienden a presentar un incremento en la ingesta relacionado con emociones negativas, a diferencia de las personas con peso normal o bajo.

De hecho, se ha planteado que existe una tendencia a regular estados emocionales negativos a través del consumo poco saludable de alimentos (Mantaua, Hattulab, \& Bornemannb, 2018). Sin embargo, no solo las emociones negativas, sino también, las positivas pueden estar relacionadas con la ingesta. Sobre todo si la intensidad con la que se presentan es elevada (Litwin, Goldbacher, Cardaciotto, \& Gambrel, 2016; Evers, Adriaanse, de Ridder \& de Witt Huberts, 2013). Particularmente, los individuos que tienen a restringir el consumo de alimentos, son más vulnerables a que sus patrones de alimentación se alteren ante las emociones, ya sean negativas o positivas (Eversa, Dingemansb, Junghansa, \& Boevéc, 2018).

Adicionalmente, se deben considerar otros factores que inciden en el consumo de alimentos como las situaciones sociales, el momento y las situaciones en las que se presenta la comida, pues todos ellos pueden modelar la conducta de ingesta (Horgana, Scalcob, Craigc, Whybrowb, \& Macdiarmidb, 2019). Por otra parte, la evaluación placentera o aversiva que se hace respecto de los alimentos y las situaciones asociadas con la conducta de ingesta, pueden propiciar un aumento o una disminución en el consumo. Por ejemplo, el placer asociado con la comida puede estimular un comer "no-homeostático", el cual, dependiendo de su frecuencia, puede llegar a ocasionar aumentos considerables en el peso corporal (Mela, 2006).

Sin embargo, la mayoría de los instrumentos de evaluación diseñados para estudiar la conducta alimentaria están centrados en aspectos patológicos relativos a la anorexia, la bulimia y el comer compulsivo (Berg, Peterson, Frazier, \& Crow, 2012; Franco, Álvarez, \& Ramírez, 2011; Garner \& Garfinkel, 1979; Garner, Olmstead, \& Polivy, 1983; Rosen, Jones, Ramírez, \& Waxman, 1996; Thompson, 2001). Si bien esos instrumentos permiten evaluar poblaciones con determinadas características, suelen resultar de poca utilidad para 
indagar las creencias y las emociones asociadas con los comportamientos alimentarios no patológicos que presenta la población general.

Por lo anterior, el objetivo general de esta investigación fue construir y valorar las propiedades psicométricas de un instrumento que permita operacionalizar las emociones y las creencias que elicitan el consumo de alimentos. Para así, proporcionar un instrumento que provea una herramienta para evaluar población sin una patología alimentaria.

Los objetivos específicos de este trabajo fueron: 1) Probar la validez de contenido por medio del análisis de jueces expertos y el piloteo; 2) Descartar los reactivos que no funcionan observando la distribución de los puntajes, capacidad de discriminación y la relación con el puntaje total de cada uno de los reactivos; 3) Probar la validez de constructo por medio de un Análisis Factorial Confirmatorio; 4) Indagar las diferencias en las puntuaciones de las dimensiones para su uso entre hombres y mujeres; y 5) Obtener puntuaciones normalizadas del instrumento para cada sexo.

El uso de este instrumento permitiría comprender mejor los motivos que subyacen a la sobre o sub ingesta en adolescentes, quienes podrían estar basando sus patrones de alimentación en creencias erróneas o cuya conducta alimentaria pudiera presentarse como una forma equivocada de regulación emocional. Por lo que es una herramienta que aporta al campo de la Psicología y de la Nutrición.

Además, es un estudio novedoso, pues los instrumentos previos suelen abordar el aspecto patológico de la alimentación, como se mencionó previamente, o bien evalúan los hábitos alimentarios de los jóvenes. Pero este instrumento se aproxima al aspecto emocional y a las creencias que motivan la alimentación sin adentrarse al proceso de enfermedad.

\section{Método}

\section{Participantes}

En el jueceo entre expertos participaron un grupo de 12 jueces expertos en el área de psicología, salud y alimentación. Mientras que, en el piloto colaboraron 15 jóvenes de entre 14 y 18 años que estudiaban el nivel medio superior en escuelas públicas de la Ciudad de México.

Finalmente, para el análisis de los reactivos por medio de muestreo no probabilístico e intencional, participaron 1833 adolescentes (918 mujeres y 915 hombres) con edades entre 14 y 18 años $(M=15.43, D T=.93)$. El 95.4\% de los participantes se encontraban estudiando el nivel medio superior, y el $4.6 \%$ iniciaba sus estudios de nivel superior en escuelas públicas de la Ciudad de México.

\section{Instrumentos}

El instrumento se creó ex profeso para esta investigación. Inicialmente el instrumento estaba conformado por 50 reactivos, con cuatro opciones de respuesta en una escala tipo Likert ( 1 = Nunca, $2=$ Algunas veces, $3=$ Con frecuencia, $4=$ Siempre $)$, distribuidos en cinco dimensiones: creencias erróneas acerca del control del peso corporal, comer emocional, desinhibición situacional, experimentación de emociones agradables al comer, e importancia que se le otorga al peso corporal. 
Tras el análisis, el instrumento final quedó conformado por 16 reactivos distribuidos en tres dimensiones: creencias erróneas acerca del control del peso corporal, formada por seis reactivos; experimentación de emociones agradables al comer e importancia que se le otorga al peso corporal, constituidas por cinco reactivos cada una (véase tabla 1). De acuerdo con lo observado, estas presentan valores de confiabilidad y validez adecuada. La especificación de las propiedades del instrumento y sus reactivos se describen más adelante en el texto.

\section{Procedimiento}

Se llevó a cabo un estudio psicométrico desde la Teoría Clásica de los Test (Muñiz, 2010), por lo que, inicialmente, se construyeron un total de 50 reactivos distribuidos en cinco dimensiones de acuerdo con la literatura: a) creencias erróneas acerca del control del peso corporal, b) experimentación de emociones agradables al comer, c) comer emocional, d) desinhibición situacional, e) e importancia otorgada al peso corporal.

Las preguntas fueron sometidas a una evaluación de jueces expertos, a quienes se les pidió valorar si los reactivos pertenecían a las dimensiones propuestas y si eran lo suficientemente claros y útiles para la población de estudio. Posteriormente, se llevó a cabo el piloto de los reactivos en un grupo de jóvenes con el objetivo de conocer si había redacción ambigua o confusa en los reactivos.

La evaluación de los expertos y el piloteo permitieron descartar 25 reactivos que presentaron bajo grado de acuerdo entre los jueces o que fueron considerados como poco claros en el piloteo. Con los 25 reactivos restantes se conformó el modelo teórico del Cuestionario de Emociones y Creencias acerca de la Alimentación y el Peso (CECAP) (tabla 1), el cual contenía las cinco dimensiones previamente mencionadas.

Finalmente, para el análisis de los reactivos, el instrumento se aplicó por estudiantes de psicología a los 1833 participantes en sus centros escolares en la Ciudad de México, de manera grupal y en horario de clases. Previo consentimiento informado de las autoridades escolares y de los propios participantes, a quienes se les pidió firmar una carta avalada por los responsables de los planteles educativos. Antes de responder el instrumento, se les informó sobre los objetivos y procedimientos del estudio, asegurándoles el carácter anónimo y confidencial de los datos. El tiempo empleado para responder el instrumento fue entre 7 y 10 minutos. Los adolescentes no tuvieron beneficios directos por su participación.

\section{Estrategia de análisis}

Se exploró la distribución de respuestas para cada reactivo, después se analizó la discriminación del reactivo y se probaron las correlaciones ítem-total. Con los reactivos que pasaron los análisis anteriores se realizó un Análisis Factorial Confirmatorio (AFC) para probar validez de constructo y se obtuvo la consistencia interna de cada factor mediante el $\alpha$. Por último, se buscaron diferencias entre hombres y mujeres en las dimensiones por medio de la $t$ de Student para muestras independientes y se obtuvieron las puntuaciones estandarizadas para cada sexo. 


\section{Resultados}

El primer paso del análisis fue explorar la distribución de las respuestas en cada uno de los reactivos para descartar aquellos que tuvieran un sesgo o curtosis altos, por lo que se descartaron aquellos que tuvieran valores entre -3 y +3 . Lo cual ocurrió con los reactivos "Aunque comer mucho me hace sentir mal, no puedo parar" (sesgo $=2.64$, curtosis $=$ 6.82), "Como más de lo que quisiera para evitar comentarios de la gente" (sesgo $=2.43$, curtosis $=5.77$ ), "Como a escondidas" (sesgo $=2.81$, curtosis $=8.43)$, "Gano peso hasta por tomar agua" ( sesgo $=3.47$, curtosis $=12.51$ ) y "Noto que cuando me siento enojado(a) o irritable, busco algo para comer" (sesgo $=2.21$, curtosis $=4.62)$.

Eliminar estos reactivos ocasionó que, tanto los factores desinhibición situacional y comer emocional, quedaran conformados por únicamente dos reactivos respectivamente. Al ya no poder ser considerados como factores, en los siguientes análisis se trabajó únicamente con tres dimensiones: a) creencias erróneas acerca del control de peso, b) experimentación de emociones agradables al comer y c) importancia que se le otorga al peso corporal (tabla 1). Posteriormente, con solo tres dimensiones y 16 reactivos, se probó la discriminación de los reactivos usando grupos extremos. Los resultados muestran que todos los reactivos probados permiten discriminar entre los grupos, por lo cual no se descartaron reactivos en este paso (tabla 2).

Después, se revisaron los valores de las correlaciones entre cada reactivo y el puntaje total del factor al que cada reactivo correspondía teóricamente. En todos los casos se obtuvieron coeficientes $\geq .30$, por lo cual, no se eliminaron reactivos en este paso del análisis (tabla 2). Posteriormente, se obtuvieron las propiedades psicométricas del instrumento. Para probar la validez de constructo, se realizó un AFC categórico, debido a que se recomienda para reactivo con menos de cinco opciones de respuesta (Flora \& Curran, 2004). La $\chi^{2}$ fue significativa $\chi^{2}(101)=1131.316, p<.001$, resultados que pueden deberse al tamaño de la muestra (Morata, Holgado, Barbero, \& Méndez, 2015).

El ajuste del modelo se evaluó con el CFI y el RMSEA. Un buen ajuste es indicado por un CFI mayor o igual a .95 y un RMSEA menor o igual a .08 (Ruiz, Pardo, \& San Martín, 2010). En este caso, el CFI fue de .973 y el RMSEA .075, indicando así un buen ajuste del modelo y, por lo tanto, adecuada validez de constructo. Las cargas factoriales, errores estándar y correlaciones entre factores se presentan en la Figura 1.

La confiabilidad se probó con el $\alpha$, los tres factores mostraron adecuados niveles de confiabilidad (tabla 2). Se obtuvieron diferencias entre hombres y mujeres en cada uno de los factores, ya que suele haber diferencias entre variables asociadas a la alimentación y el peso entre sexos (Ramos et al; 2016); y se observó que las mujeres presentaron mayores niveles de creencias erróneas acerca del control de peso y de importancia que le otorgan al peso corporal, pero no hubo diferencias entre sexos respecto a la experimentación de emociones agradables al comer (tabla 3).

Finalmente, se calcularon las puntuaciones $T$ para cada dimensión. Debido a las diferencias de magnitud mediana que se observaron entre sexos, se obtuvieron dichas puntuaciones estandarizadas para hombres y mujeres por separado (tabla 4). 
Tabla 1

Modelo teórico

\begin{tabular}{|c|c|}
\hline Dimensiones & Reactivos \\
\hline \multirow{7}{*}{$\begin{array}{l}\text { Creencias } \\
\text { erróneas acerca } \\
\text { del control del } \\
\text { peso corporal }\end{array}$} & Pienso que todos los alimentos engordan \\
\hline & Pienso que haga lo que haga, engordo \\
\hline & Pienso que aunque como poco, subo de peso \\
\hline & Pienso que aunque como poco, engordo \\
\hline & Pienso que tiendo a ganar peso con mayor facilidad que otros \\
\hline & Aunque coma saludablemente, gano peso \\
\hline & Gano peso basta por tomar agua \\
\hline \multirow{4}{*}{ Comer emocional } & Noto que cuando me siento triste, busco algo para comer \\
\hline & Noto que cuando me siento solo(a), busco algo para comer \\
\hline & Aunque comer mucho me bace sentir mal, no puedo parar \\
\hline & Noto que cuando me siento enojado(a) o irritable, busco algo para comer \\
\hline \multirow{4}{*}{$\begin{array}{l}\text { Desinbibición } \\
\text { situacional }\end{array}$} & Como cuando otros están comiendo aunque no tenga hambre \\
\hline & Como todo lo que me sirven aunque me sienta satisfecho(a) \\
\hline & Como más de lo que quisiera para evitar comentarios de la gente \\
\hline & Como a escondidas \\
\hline \multirow{5}{*}{$\begin{array}{l}\text { Experimentación } \\
\text { de emociones } \\
\text { agradables } \\
\text { al comer }\end{array}$} & Comer es un placer \\
\hline & Comer me hace feliz \\
\hline & Disfruto comer \\
\hline & Las horas de la comida son momentos agradables \\
\hline & Comer me hace sentir tranquilo(a) \\
\hline \multirow{5}{*}{$\begin{array}{l}\text { Importancia que } \\
\text { se le otorga al } \\
\text { peso corporal }\end{array}$} & Pienso que engordar sería lo peor que me podría pasar \\
\hline & Pienso que el peso de las personas es importante \\
\hline & Cuando como de más me siento culpable \\
\hline & Me fijo en el peso de las personas \\
\hline & Creo que debiera hacer una dieta \\
\hline
\end{tabular}

Nota. En itálica aparecen aquellos reactivos y factores que fueron descartados tras analizar la distribución de los datos. 


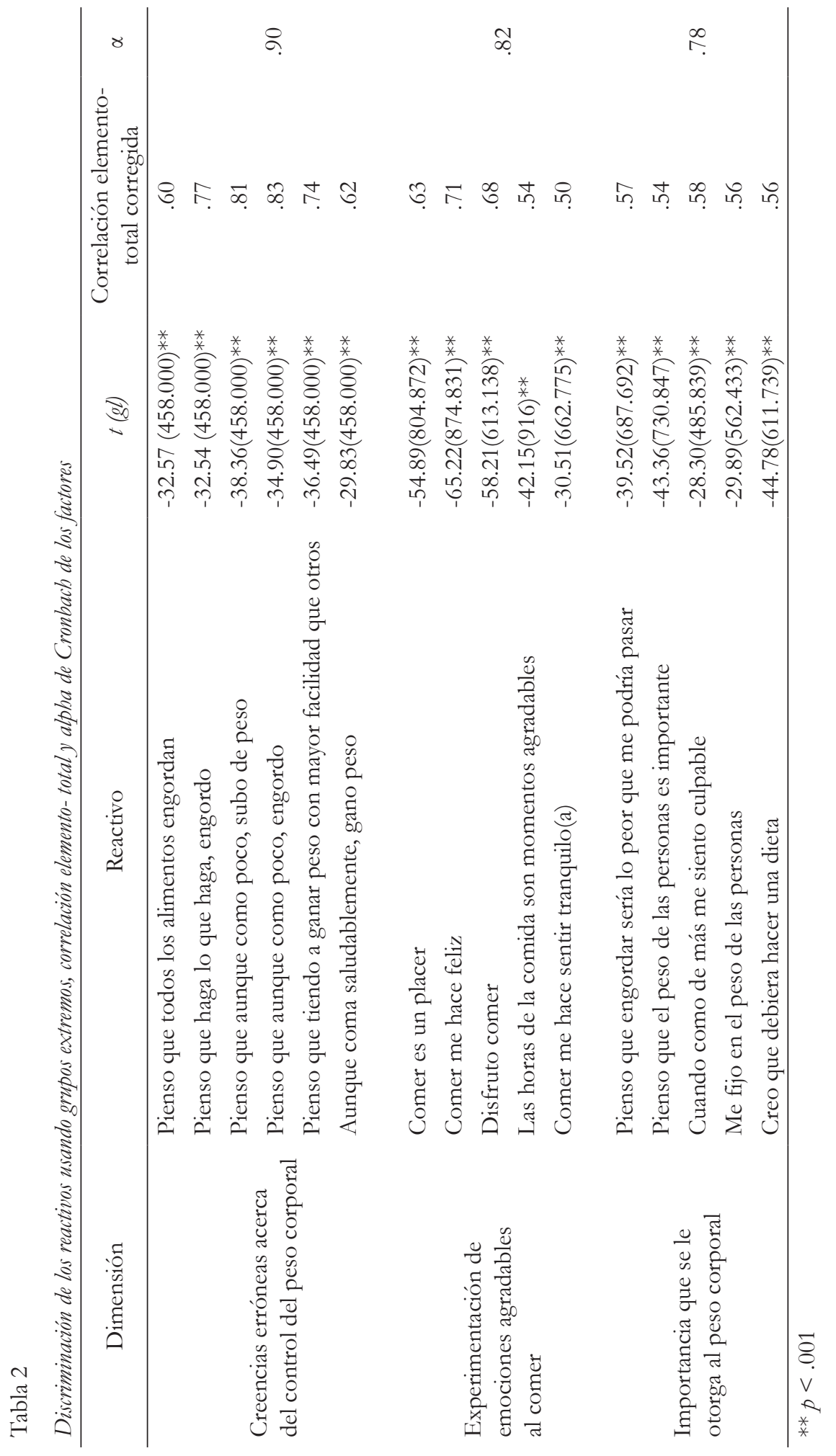

Actualidades en Psicología, 33(127), julio-diciembre 2019, 37-48 
Tabla 3

Contraste entre hombres y mujeres en los factores del CECAP

\begin{tabular}{|c|c|c|c|c|c|c|c|c|}
\hline \multirow[b]{2}{*}{ Factor } & \multicolumn{2}{|c|}{ Hombres } & \multicolumn{2}{|c|}{ Mujeres } & \multirow[b]{2}{*}{$t(g l)$} & \multicolumn{2}{|c|}{$95 \% I C$} & \multirow[b]{2}{*}{$\begin{array}{l}d \text { de } \\
\text { Cohen }\end{array}$} \\
\hline & $\bar{X}$ & DT & $\bar{X}$ & DT & & LI & LS & \\
\hline $\begin{array}{l}\text { Creencias erróneas } \\
\text { acerca del control } \\
\text { del peso corporal }\end{array}$ & 7.62 & 2.63 & 10.12 & 4.46 & $\begin{array}{c}14.57 \\
(1489.13)^{* *}\end{array}$ & 2.16 & 2.83 & -.68 \\
\hline $\begin{array}{l}\text { Experimentación } \\
\text { de emociones } \\
\text { agradables al comer }\end{array}$ & 13.58 & 3.65 & 13.47 & 3.54 & $\begin{array}{l}-.646 \\
(1831)\end{array}$ & -.43 & .22 & .03 \\
\hline $\begin{array}{l}\text { Importancia que se le } \\
\text { otorga al peso corporal }\end{array}$ & 8.57 & 2.96 & 10.50 & 3.74 & $\begin{array}{c}12.21 \\
(1741.01)^{* *}\end{array}$ & 1.61 & 2.23 & -.57 \\
\hline
\end{tabular}

Nota. IC $=$ intervalo de confianza, LI = límite inferior, LS = límite superior.

$* * p<.001$
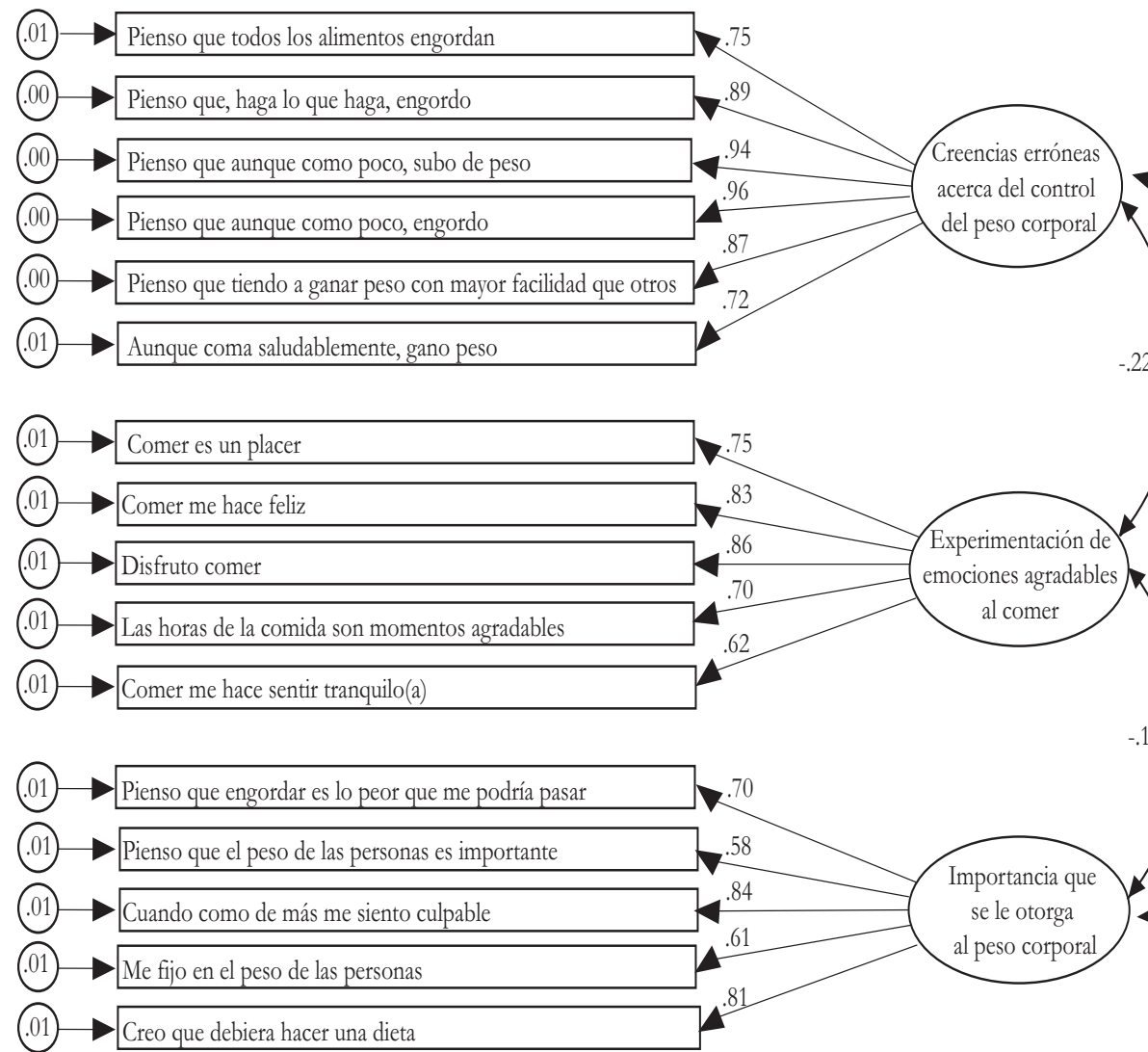

Figura 1. Análisis Factorial Confirmatorio del CECAP 
Tabla 4

Puntuaciones t de las dimensiones del CECAP

\begin{tabular}{|c|c|c|c|c|c|c|c|}
\hline \multirow{3}{*}{$\begin{array}{c}\text { Puntuación } \\
\text { natural }\end{array}$} & \multicolumn{6}{|c|}{ Puntuaciones $t(M=50, D T=10)$} & \multirow{3}{*}{$\begin{array}{c}\text { Puntuación } \\
\text { natural }\end{array}$} \\
\hline & \multicolumn{2}{|c|}{$\begin{array}{l}\text { Creencias erróneas } \\
\text { acerca del control } \\
\text { del peso corporal }\end{array}$} & \multicolumn{2}{|c|}{$\begin{array}{l}\text { Experimentación } \\
\text { de emociones } \\
\text { agradables al comer }\end{array}$} & \multicolumn{2}{|c|}{$\begin{array}{l}\text { Importancia que } \\
\text { se le otorga al } \\
\text { peso corporal }\end{array}$} & \\
\hline & Mujeres & Hombres & Mujeres & Hombres & Mujeres & Hombres & \\
\hline 5 & & & 26 & 26 & 35 & 38 & 5 \\
\hline 6 & 41 & 44 & 29 & 29 & 38 & 41 & 6 \\
\hline 7 & 43 & 48 & 32 & 32 & 41 & 45 & 7 \\
\hline 8 & 45 & 51 & 35 & 35 & 43 & 48 & 8 \\
\hline 9 & 47 & 55 & 37 & 37 & 46 & 51 & 9 \\
\hline 10 & 50 & 59 & 40 & 40 & 49 & 55 & 10 \\
\hline 11 & 52 & 63 & 43 & 43 & 51 & 58 & 11 \\
\hline 12 & 54 & 67 & 46 & 46 & 54 & 62 & 12 \\
\hline 13 & 56 & 70 & 49 & 48 & 57 & 65 & 13 \\
\hline 14 & 59 & 74 & 51 & 51 & 59 & 68 & 14 \\
\hline 15 & 61 & 78 & 54 & 54 & 62 & 72 & 15 \\
\hline 16 & 63 & 82 & 57 & 57 & 65 & 75 & 16 \\
\hline 17 & 65 & 86 & 60 & 59 & 67 & 78 & 17 \\
\hline 18 & 68 & 89 & 63 & 62 & 70 & 82 & 18 \\
\hline 19 & 70 & 93 & 66 & 65 & 73 & 85 & 19 \\
\hline 20 & 72 & 97 & 68 & 68 & 75 & 89 & 20 \\
\hline 21 & 74 & 101 & & & & & 21 \\
\hline 22 & 77 & 105 & & & & & 22 \\
\hline 23 & 79 & 108 & & & & & 23 \\
\hline 24 & 81 & 112 & & & & & 24 \\
\hline
\end{tabular}

\section{Discusión}

Este estudio se generó a partir de la necesidad de contar con un instrumento confiable y válido para evaluar las emociones y creencias que tienen las personas acerca de la alimentación y el peso corporal, debido a que la mayoría de los instrumentos en este campo se centran en las patologías de la conducta alimentaria. Los datos obtenidos indican que el instrumento es confiable para evaluar tres dimensiones: creencias erróneas acerca del control del peso, experimentación de emociones agradables al comer, e importancia que se le otorga al peso corporal.

Asimismo, el AFC mostró un buen ajuste y, por lo tanto, una adecuada validez de constructo (AERA, APA, \& NCME, 1999). Además, las comparaciones entre hombres y mujeres, arrojaron la tendencia también esperada de acuerdo con la literatura: las mujeres 
mostraron más creencias negativas acerca del control del peso corporal y le otorgan mayor importancia al peso corporal; mientras que, tanto hombres como mujeres mostraron niveles similares de emociones agradables al momento de comer.

Si las dietas o la elección de alimentos se basa en las creencias erróneas acerca del control de peso y en la importancia que se le otorga al peso corporal, es posible que se estén generando patrones alimentarios poco saludables, compuestos también por alimentos que generan emociones agradables. Estos podrían, por ejemplo, ser altos en carbohidratos (Benton, 2002), al basar la elección en las emociones evocadas por alimentos específicos (Gutjar et al; 2015), se podría presentar un consumo bajo de alimentos pertenecientes a distintos grupos. Por lo que valdría la pena investigar las emociones y creencias acerca de la alimentación y el peso en relación con la dieta, la condición nutricia, y los índices de masa corporal de distintas poblaciones.

Para ello, en estudios posteriores, será necesario probar el funcionamiento del instrumento en otros grupos etarios con el fin de iniciar su uso para la investigación en diferentes contextos. Asimismo, es pertinente redactar y probar reactivos que evalúen las otras dos dimensiones que, inicialmente se tenían contempladas para este instrumento: comer emocional y desinhibición situacional. Además, es importante tener más evidencias de las propiedades psicométricas del instrumento por medio de otros métodos o la validez de criterio entre otros.

\section{Referencias}

Adriaanse, M., De Ridder, D., \& Evers, C. (2011). Emotional eating: Eating when emotional or emotional about eating? Psychology and Health, 26(1), 23-39.

AERA, APA, \& NCME (1999). Standards for educational and psychological testing. Washington: AERA.

Benton, D. (2002). Carbohydrate ingestion, blood glucose and mood. Neuroscience and biobehavioral reviews, 26(3), 293-308.

Berg, K. C., Peterson, C. B., Frazier, P., \& Crow, S. J. (2012). Psychometric evaluation of the Eating Disorder Examination and Eating Disorder Examination - Questionnaire: A systematic review of the literature. International Journal of Eating Disorders, 45(3), 428-438.

Bublitz, M. G., Peracchio, L. A., \& Block, L. G. (2010). Why did I eat that? Perspectives on food decision making and dietary restraint. Journal of Consumer Psychology, 20(3), 239-258. doi: 10.1016/j.jcps.2010.06.008

Cruwys, T., Bevelander, K. E., \& Hermans, R. C. J. (2015). Social modeling of eating: A review of when and why social influence affects food intake and choice. Appetite, 86, 3-18. doi: 10.1016/j.appet.2014.08.035

DeJesusa, J., Gelmana S., Viechnickig, B., Appugliesec, D., Millerd, A., Rosenblume, K., ... \& Lumengb, J. (2018). An investigation of maternal food intake and maternal food talk as predictors of child food intake. Appettite, 127, 356-363. doi:10.1016/j.appet.2018.04.018 
Evers, C., Adriaanse, M., de Ridder, D., \& de Witt Huberts, J. (2013). Research report Good mood food. Positive emotion as a neglected trigger for food intake. Appetite, 68, 1-7. doi:10.1016/j.appet.2013.04.007

Eversa, C., Dingemansb, A., Junghansa, A., \& Boevéc, A. (2018). Feeling bad or feeling good, does emotion affect your consumption of food? A meta-analysis of the experimental evidence. Neuroscience Biobehavioral Reviens, 92, 195-208. doi: /10.1016/j.neubiorev.2018.05.028

Flora, D. B. \& Curran, P. J. (2004). An empirical evaluation of alternative methods of estimation for confirmatory factor analysis with ordinal data. Psychological Methods, 9(4), 466-491. doi: 10.1037/1082-989X.9.4.466

Franco, K., Alvarez, G. L., \& Ramírez, R. E. (2011). Instrumentos para trastornos del comportamiento alimentario validados en mujeres mexicanas: una revisión de la literatura. Revista Mexicana de Trastornos Alimentarios, 2, 148-164.

Garner, D., \& Garfinkel, P. (1979). The Eating Attitudes Test: An index of the symptoms of anorexia nervosa. Psychological Medicine, 9(2), 273-279.

Garner, D. M., Olmstead, M. P., \& Polivy, J. (1983). Development and validation of a multidimensional eating disorders inventory for anorexia nervosa and bulimia. International Journal of Eating Disorders, 2(2), 15-34. doi: 10.1002/1098-108X(198321)2:2<15::AID-EAT2260020203>3.0.CO;2-6

Geraerts, E., Bernstein, D. M., Merckelbach, H., Linders, C., Raymaekers, L., \& Loftus, E. F. (2008). Lasting false beliefs and their behavioral consequences. Psychologycal Science, 19(8), 749-753. doi: 10.1111/j.1467-9280.2008.02151

Gutjar, S., de Graaf, C., Kooijman, V., de Wijk, R. A., Nys, A., ter Horst, G. J., \& Jager, G. (2015). The role of emotions in food choice and liking. Food Research International, 76(2), 216-223. doi: 10.1016/j.foodres.2014.12.022

Horgana, G. W., Scalcob, K., Craigc, T., Whybrowb, S., \& Macdiarmidb, J. I. (2019). Social, temporal and situational influences on meat consumption in the UK population. Appettite, 138, 1-9. doi:10.1016/j.appet.2019.03.007

Howe, D., Anderson, J., \& Dewhurst, A. (2017). False memories, but not false beliefs, affect implicit attitudes for food preferences. Acta Psychologica, 179, 14-22. doi:10.1016/j. actpsy.2017.07.002

Lally, P., Bartle, N., \& Wardle, J. (2011). Social norms and diet in adolescents. Appetite, 57(3), 623-627. doi: 10.1016/j.appet.2011.07.015.0

Litwin, R., Goldbacher, E. M., Cardaciotto, L. \& Gambrel, L. E. (2016). Negative emotions and emotional eating: the mediating role of experiential avoidance. Eating and Weight Disorders, 22(1), 97-104. doi: 10.1007/s40519-016-0301-9

Mantaua, A., Hattulab, S., \& Bornemannb, T. (2018). Individual determinants of emotional eating: A simultaneous investigation. Appettite, 130, 93-103. doi: 10.1016/j. appet.2018.07.015 
Mela, D. J. (2006). Eating for pleasure or just wanting to eat? Reconsidering sensory hedonic responses as a driver of obesity. Appetite, 47(1), 10-17. doi:10.1016/j. appet.2006.02.006

Morata, M. A., Holgado, F. P., Barbero, I., \& Méndez, G. (2015). Análisis Factorial Confirmatorio. Recomendaciones sobre mínimos cuadrados no ponderados en función del error tipo I de Ji cuadrado y RMSEA. Acción Psicológica, 12(1), 79-90. doi: 10.5944/ap.12.1.14362

Muñiz, J. (2010). Las teorías de los tests: Teoría clásica y teoría de respuesta a los ítems. Papeles del Psicólogo, 31(1), 57-66.

Ramos, J. H., González, K. E., \& Silva, C. (2016). Efecto de la interacción entre el sexo y el peso sobre el comer emocional en adolescentes. Revista Psicología y Salud, 26(1), 63-68.

Rodgers, R. (2016). The role of the "Healthy Weight" discourse in body image and eating concerns: An extension of sociocultural theory. Eating Behaviors, 22, 194-198. doi: 10.1016/j.eatbeh.2016.06.004 1471-0153

Rosen, J. C., Jones, A., Ramirez, E., \& Waxman, S. (1996). Body Shape Questionnaire: Studies of validity and reliability. International Journal of Eating Disorders, 20(3), 315-319. doi: 10.1002/(SICI)1098-108X(199611)20:3<315::AID-EAT11>3.0.CO;2-Z

Ruiz, M. A., Pardo, A., \& San Martín, R. (2010). Modelos de ecuaciones estructurales. Papeles del Psicólogo, 31(1), 34-45.

Sinha, J. (2016). We are where we eat: How consumption contexts induce (un)healthful eating for stigmatized overweight consumers. Journal of Consumer Psychology 26(2), 289-297. doi: 10.1016/j.jcps.2015.06.015

Thompson, J. K. (2001). Body image, eating disorders, and obesity: An integrative guide for assessment and treatment. Washington: American Psychological Association.

Van Strien, T., Donker, M., \& Ouwens, M. A. (2016). Is desire to eat in response to positive emotions an 'obese' eating style: Is Kummerspeck for some people a misnomer? Appetite, 100, 225-235. doi: 10.1016/j.appet.2016.02.035 